Full moment tensor and source location inversion based on full waveform adjoint inversion: application at the Geysers geothermal field

C. Morency, R. J. Mellors

April 2, 2012

Society of Exploration Geophysicist (SEG) Las Vegas, NV, United States November 4, 2012 through November 9, 2012 
This document was prepared as an account of work sponsored by an agency of the United States government. Neither the United States government nor Lawrence Livermore National Security, LLC, nor any of their employees makes any warranty, expressed or implied, or assumes any legal liability or responsibility for the accuracy, completeness, or usefulness of any information, apparatus, product, or process disclosed, or represents that its use would not infringe privately owned rights. Reference herein to any specific commercial product, process, or service by trade name, trademark, manufacturer, or otherwise does not necessarily constitute or imply its endorsement, recommendation, or favoring by the United States government or Lawrence Livermore National Security, LLC. The views and opinions of authors expressed herein do not necessarily state or reflect those of the United States government or Lawrence Livermore National Security, LLC, and shall not be used for advertising or product endorsement purposes. 


\title{
Full moment tensor and source location inversion based on full waveform adjoint inversion: applica- tion at the Geysers geothermal field
}

\author{
Christina Morency* and Robert J. Mellors, Lawrence Livermore National Laboratory
}

\section{SUMMARY}

Source parameters, namely full moment tensor and source location, are investigated based on a full waveform adjoint inversion. The method relies on full wave simulations and takes advantage of the misfit between observed and synthetic seismograms. An adjoint wavefield is calculated by backpropagating the difference between observed and synthetics from the receivers to the source. The interaction between this adjoint wavefield and the regular forward wavefield helps define source parameter Fréchet derivatives, that is, the sensitivity of the misfit with respect to the source parameters. We demonstrate the method on two synthetic cases before tackling a recent event recorded at the Geysers geothermal field.

\section{INTRODUCTION}

The development of high-performance computing and numerical techniques enabled global and regional tomography to reach high levels of precision, and seismic adjoint tomography has become a state-of-the-art tomographic technique (e.g., Tape et al., 2009; Peter et al., 2011). Here we focus on the determination of source parameters (full moment tensor and location) based on the same approach. The first section gives a summary of the theory. In the second section we investigate the potential of recovering source parameters using a $1 \mathrm{D}$ structural model of the Geysers area (model GIL7 by Dreger and Romanowicz, 1994). Forward and adjoint numerical simulations are performed using a spectral-element code (open source SPECFEM3D, Komatitsch et al., 2005).

\section{THEORY}

Kim et al. (2011) have recently presented a detailed analysis of adjoint centroid-moment tensor inversion with an illustration of the technique for southern California regional earthquakes. Here we aim at focusing the same approach at a local scale and ultimately at characterizing smaller events.

Centroid-moment tensor (CMT) solutions are calculated by minimizing waveform differences between observed and simulated seismograms based on an adjoint method. The nonlinear adjoint CMT inversion algorithm based on a conjugategradient method requires three simulations at each iteration

- a forward simulation to obtain the synthetics for the current source parameters,

- an adjoint simulation triggered by the injection of timereversed differences between observed and synthetics at each receivers acting as simultaneous sources,

- a forward simulation to compute the step length in the conjugate-gradient direction.
A CMT solution is defined by a moment tensor $\mathbf{M}$, a centroid location $\mathbf{x}_{S}$, and a source time function $S$. To derive the CMT source parameters of an event, we minimize the squared differences between observed seismograms (data) d recorded at a series of stations located at $\mathbf{x}_{r}$ and simulated seismograms (synthetics) $\mathbf{s}$ calculated for a preliminary source model $\mathbf{m}=$ $\mathbf{M}, \mathbf{x}_{s}, S$. Such a misfit writes as

$$
\chi=\frac{1}{2} \sum_{r p} \int w_{r p}(t)\left\|\mathbf{s}\left(\mathbf{x}_{r}, t ; \mathbf{m}\right)-\mathbf{d}\left(\mathbf{x}_{r}, t\right)\right\|^{2} \mathrm{~d} t,
$$

where data and synthetics have been filtered, and $w_{r p}$ refers to a windowing function assigned to the $p$ th window at the $r$ th receiver. Such a windowing is realized using the semi-automated open source software FLEXWIN (Maggi et al., 2009), which warrants maximizing the number of meaningful misfit measurements while avoiding seismic noise.

Let's recall that the forward wave equation writes as

$$
\rho \ddot{\mathbf{s}}=\nabla \cdot(\mathbf{c} \nabla \mathbf{s})-\mathbf{M} \cdot \nabla \delta\left(\mathbf{x}-\mathbf{x}_{S}\right) S(t) .
$$

where $\rho$ and $\mathbf{c}$ are the density and the elastic tensor, respectively. Kim et al. (2011) demonstrate that the adjoint wave equation is simply

$\rho \ddot{\mathbf{s}}^{\dagger}=\nabla \cdot\left(\mathbf{c} \nabla \mathbf{s}^{\dagger}\right)+\sum_{r p}\left[w_{r p}(t)(\mathbf{s}-\mathbf{d})\left(T-2 t_{0}-t\right) \boldsymbol{\delta}\left(\mathbf{x}-\mathbf{x}_{r}\right)\right.$

where the subscript $\dagger$ is used to define the adjoint wavefield. Note that the adjoint source is the time-reversed, windowed and filtered waveform difference between data and synthetics at each stations. Also note that except for the source, forward and adjoint wave equations (2-3) are strictly identical meaning that the same solver can be used as long as the source is properly defined.

Based on the expression of the variation of $\chi$ and the linearized expression of the variation of equations (2-3) with respect to the source parameters, the Fréchet derivatives for the moment tensor $\mathbf{M}$ and the centroid location $\mathbf{x}_{S}$ can be expressed as

$$
\begin{gathered}
\frac{\partial \chi}{\partial M_{i j}}=\int \varepsilon_{i j}^{\dagger} S\left(T-2 t_{0}-t\right) \mathrm{d} t, \\
\frac{\partial \chi}{\partial x_{i}^{s}}=\int \partial_{x_{i}^{s}}\left[\mathbf{M}: \varepsilon^{\dagger}\right] S\left(T-2 t_{0}-t\right) \mathrm{d} t,
\end{gathered}
$$

where $\varepsilon^{\dagger}=\frac{1}{2}\left[\nabla \mathbf{s}^{\dagger}+\left(\nabla \mathbf{s}^{\dagger}\right)^{T}\right]$ is the adjoint strain tensor. Note that similar Fréchet derivatives for the source time function can be derived (see Kim et al., 2011, for details).

It is interesting to note that contrary to the structural Fréchet derivatives (e.g., Tromp et al., 2005; Morency et al., 2009), the source parameter Fréchet derivatives are only function of the adjoint wavefield, whereas the formers result of the interaction between forward and adjoint wavefields. This means that to 


\section{Full moment tensor inversion}

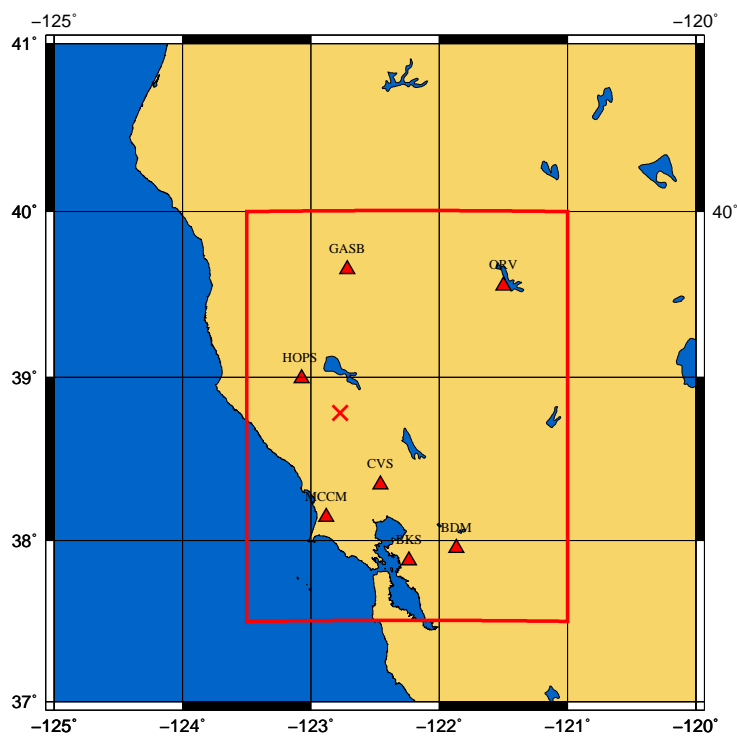

Figure 1: The red rectangle represents the numerical domain investigated, which corresponds to a $225 \mathrm{~km} \times 280 \mathrm{~km} \times 36 \mathrm{~km}$ box. The red triangles refer to the recording stations and the cross gives the location of the source studied (a synthetic event and an event from the Northern California Earthquake Data Center (NCEDC) catalog).

calculate source parameter Fréchet derivatives, one does not need to have stored or to recalculate on the fly the forward wavefield when performing the adjoint calculation and constructing the kernels.

Once the Fréchet derivatives calculated, the key is to use the information they carry in terms of the variation of the misfit function with respect to the source parameters, to iteratively reduce this misfit and thereby constrain the source parameters. To do so we use a conjugate-gradient algorithm.

\section{APPLICATION}

In the following we illustrate the technique with synthetic and real cases. Notice that in all cases the signals are filtered, and preprocessing also includes removing the instrument response for the real case.

\section{Synthetic cases}

Using the GIL7 1D velocity model, we generate synthetic observed and modeled seismograms based on a known CMT solution and an erroneous CMT solution, respectively. Waveforms are recorded at the stations BDM, BKS, CVS, GASB, HOPS, MCCM and ORV from the Berkeley (BK) network displayed in Figure 1. The goal is then to recover the accurate known CMT solution, starting with the erroneous CMT solution: case (a) only the moment tensor coefficients are wrong,
Table 1: Focal mechanism, centroid location, and moment tensor components for the synthetic cases.

\begin{tabular}{|l|c|cc|c|}
\hline & $\begin{array}{c}\text { initial } \\
\mathrm{m}_{00}\end{array}$ & $\begin{array}{c}\text { case (a) } \\
\mathrm{m}_{06}\end{array}$ & $\begin{array}{c}\text { case (b) } \\
\mathrm{m}_{12}\end{array}$ & solution \\
& & & & \\
& & & & \\
& & & & \\
Mw (-) & 4.27 & 4.27 & 4.27 & 4.27 \\
moment & & 3.25 & 3.20 & 3.22 \\
latitude $\left(^{0}\right)$ & 3.22 & 38.7823 & 38.7823 & 38.7823 \\
longitude $\left(^{0}\right)$ & -122.7715 & -122.7715 & -122.7715 & -122.7715 \\
depth $(\mathrm{km})$ & $4.68^{a} / 6.00^{b}$ & 4.68 & 4.53 & 4.68 \\
$\mathrm{Mrr}^{\ddagger}$ & -0.5446 & -0.5676 & -0.5464 & -0.5446 \\
$\mathrm{Mtt}^{\ddagger}$ & 1.0869 & -0.5208 & -0.8061 & -0.5423 \\
$\mathrm{Mpp}^{\ddagger}$ & -0.5423 & 1.0592 & 1.3594 & 1.0859 \\
$\mathrm{Mrt}^{\ddagger}$ & -0.4956 & -0.8680 & -0.7131 & -0.7289 \\
$\mathrm{Mrp}^{\ddagger}$ & -0.7289 & -0.4854 & -0.4661 & -0.4956 \\
$\mathrm{Mtp}^{\ddagger}$ & 2.9528 & 2.9565 & 2.8267 & 2.9528 \\
\hline
\end{tabular}

* Scalar moment and moment tensor coefficients unit is $10^{22}$ dyne.cm

and case (b) moment tensor coefficients and depth are wrong.

The results of the iterative inversions are displayed in Fig. 2, and show an excellent convergence to the exact solution in 6 and 12 iterations for the case (a) and (b), respectively. The misfit reduction corresponding to case (a) and (b) is of $99 \%$ and $97 \%$, respectively, indicating a proper station azimuthal coverage to resolve events in the area. Table 1 offers a closer look at the source parameters for the initial erroneous model $\mathrm{m}_{00}$, the final iterative solution for case (a) $\mathrm{m}_{06}$ and (b) $\mathrm{m}_{12}$, and the exact solution. Finally, in Fig. 3 we display a comparison of the 3-component seismograms recorded at the station BDM between the "data", the initial model $\mathrm{m}_{00}$, and the inverted model $\mathrm{m}_{06}$. Signals have been filtered between 20 and $50 \mathrm{~s}$. One can appreciate the improvement of the fit between "data" and synthetics. Similar improvement in fitting to "data" is observed when performing the 7 parameter inversion. Note that in that case, in order to resolve the depth, the signals are filtered between 2 and $8 \mathrm{~s}$.

\section{January 4th 2009 event at the Geysers, NCEDC ID 51214595}

In this section, we present a source parameter inversion for an event which took place on January 4th 2009 at the Geysers. We use data from the NCEDC recorded at the stations BDM, BKS, CVS, GASB, HOPS, MCCM and ORV (displayed in Figure 1). The initial source solution $m_{00}$ is a deviatoric solution from the NCEDC catalog (see Table 2). A good correspondence between data and synthetics is found when filtering between 20 and $50 \mathrm{~s}$. These periods are relatively insensitive to the details of the crustal structure, making the use of a 1D model adequate (e.g., Pasyanos et al., 1996). This yields to a weak sensitivity to depth, which cannot be accurately recovered. We therefore focus on inverting only the moment tensor for now. 

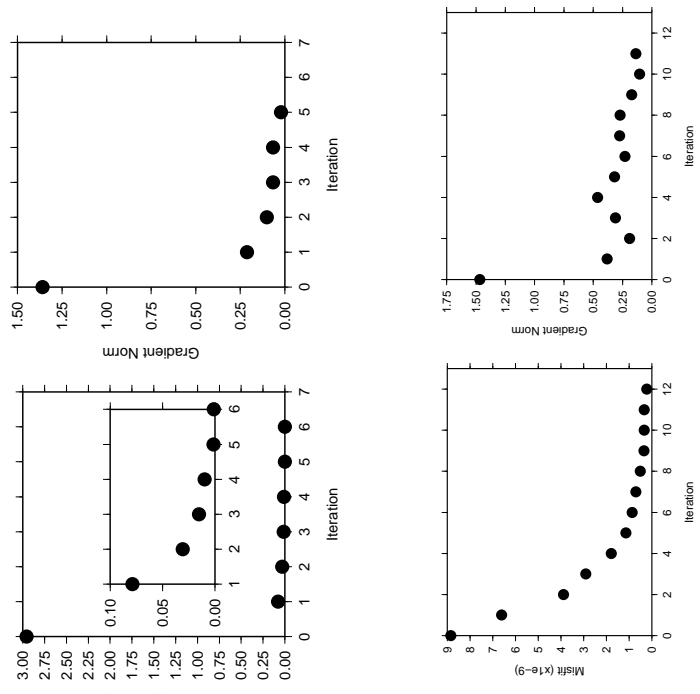

$(1+-\partial \mid x)$ น
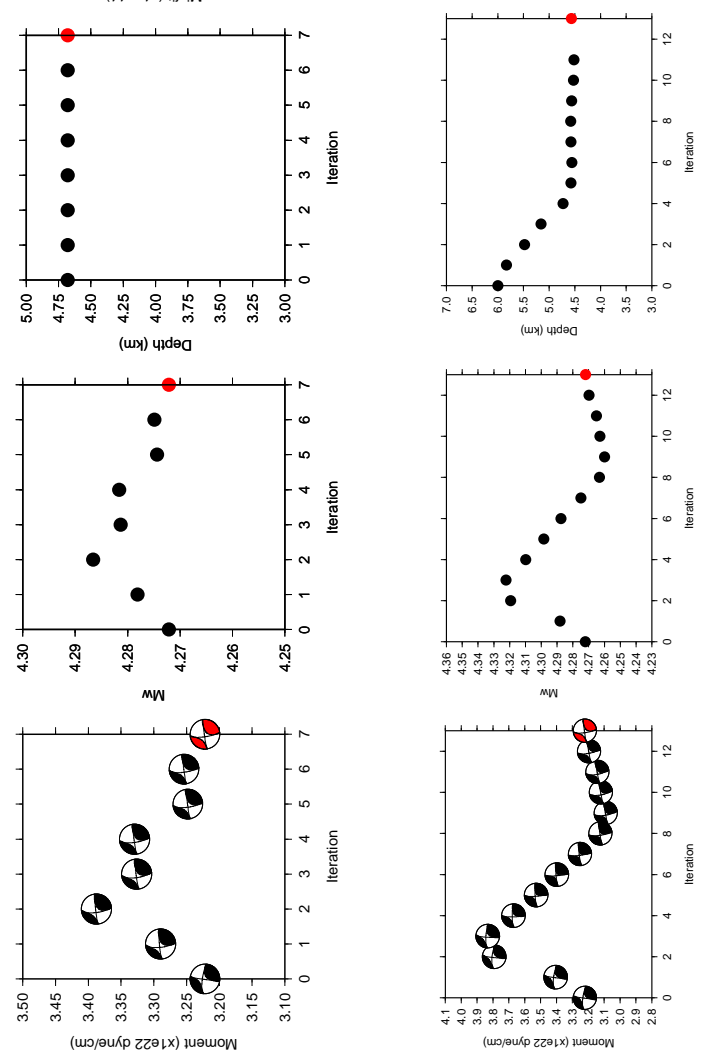

(a)
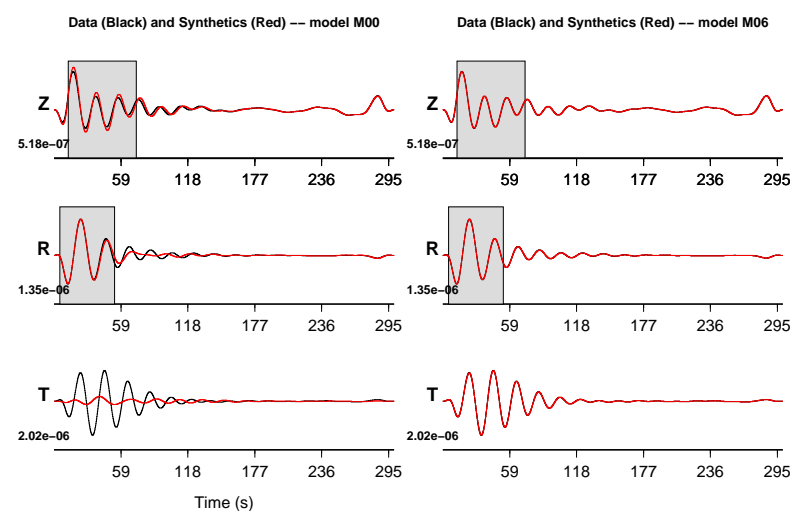

Figure 3: Synthetic case: comparison of the 3-component seismograms at station BDM between "data" (black) and initial model $\mathrm{m}_{00}$ and final models $\mathrm{m}_{06}$ for the 6 parameter inversion. Signals have been filtered between 20 and $50 \mathrm{~s}$. The shaded rectangles correspond to the windows picked by FLEXWIN where waveform measurements used during the inversion are calculated. [Z: vertical, R: radial and T: transversal]

Results of the iteration are displayed in Fig. 4. One can see that contrary to the synthetic cases performed with a fully constrained velocity model, the misfit reaches a plateau at a non zero values. The misfit reduction in that case is of $47 \%$. The final model is the best solution that can be reached with the $1 \mathrm{D}$ velocity model used and the source location determined from the NCEDC catalog. The improvement of the fitting between data and synthetic 3-component seismograms is displayed in Fig. 5. Remaining differences can be in part attributed to the fact that 3D heterogeneities in the crust are not well accounted for.

\section{CONCLUSIONS}

We use a full waveform adjoint method to investigate the inversion for source parameters, namely full moment tensor and source location, at the Geysers geothermal field. The event considered here is of magnitude 4+, which allows to use low frequency signals and a simple 1D velocity model. We note that in that case, depth resolution is weak. To improve this latter, one needs to use a more accurate $3 \mathrm{D}$ velocity model to account for 3D heterogeneities in the crust. Nevertheless, we were able to invert for the moment tensor coefficients. The future work and strategy is to improve the 1D velocity model by realizing a local adjoint tomography, to be able to invert for depth. By performing a high frequency 3D adjoint tomography using the dense Calpine/Unocal network and dataset available through the NCEDC website, the resulting $3 \mathrm{D}$ velocity model would allow to resolve even smaller events.

parameters (moment tensor coefficients) when the location is known, and column (b) presents the iterative inversion for 7 parameters (moment tensor coefficients and depth). The exact solution is in red. Each panel displays the scalar moment, magnitude, depth, misfit, and norm of the gradient, from bottom to top.

\section{ACKNOWLEDGMENTS}

This work was performed under the auspices of the U.S. Department of Energy by Lawrence Livermore National Laboratory under Contract DE-AC52-07NA27344. 


\section{Full moment tensor inversion}

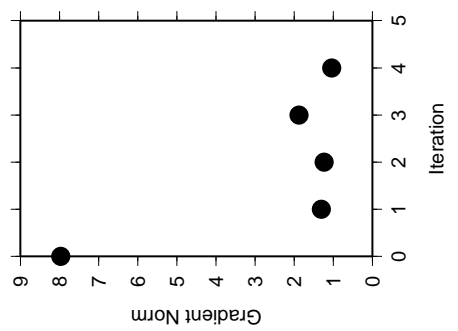

Table 2: Focal mechanism, centroid location, and moment tensor components for the January 4th 2009 event at the Geysers. The initial CMT solution corresponds to the deviatoric solution from the NCEDC catalog.

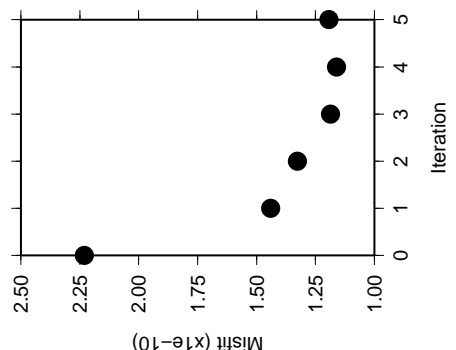

\begin{tabular}{|l|c|c|}
\hline & $\begin{array}{c}\text { initial } \\
\mathrm{m}_{00}\end{array}$ & $\begin{array}{c}\text { final model } \\
\mathrm{m}_{05}\end{array}$ \\
\hline & & \\
Mw (-) & 4.27 & 4.54 \\
moment $^{\ddagger}$ & 3.22 & 8.23 \\
latitude $\left(^{0}\right)$ & 38.7823 & 38.7823 \\
longitude $\left(^{0}\right)$ & -122.7715 & -122.7715 \\
depth $(\mathrm{km})$ & 4.68 & 4.68 \\
Mrt & -0.5446 & -0.7175 \\
$\mathrm{Mtt}^{\ddagger}$ & -0.5423 & 0.3137 \\
$\mathrm{Mpp}^{\ddagger}$ & 1.0869 & 1.5910 \\
$\mathrm{Mrt}^{\ddagger}$ & -0.7289 & -1.841 \\
$\mathrm{Mrp}^{\ddagger}$ & -0.4956 & -5.7520 \\
$\mathrm{Mtp}^{\ddagger}$ & 2.9528 & 5.4476 \\
\hline
\end{tabular}

* Scalar moment and moment tensor coefficients unit is $10^{22}$ dyne.cm
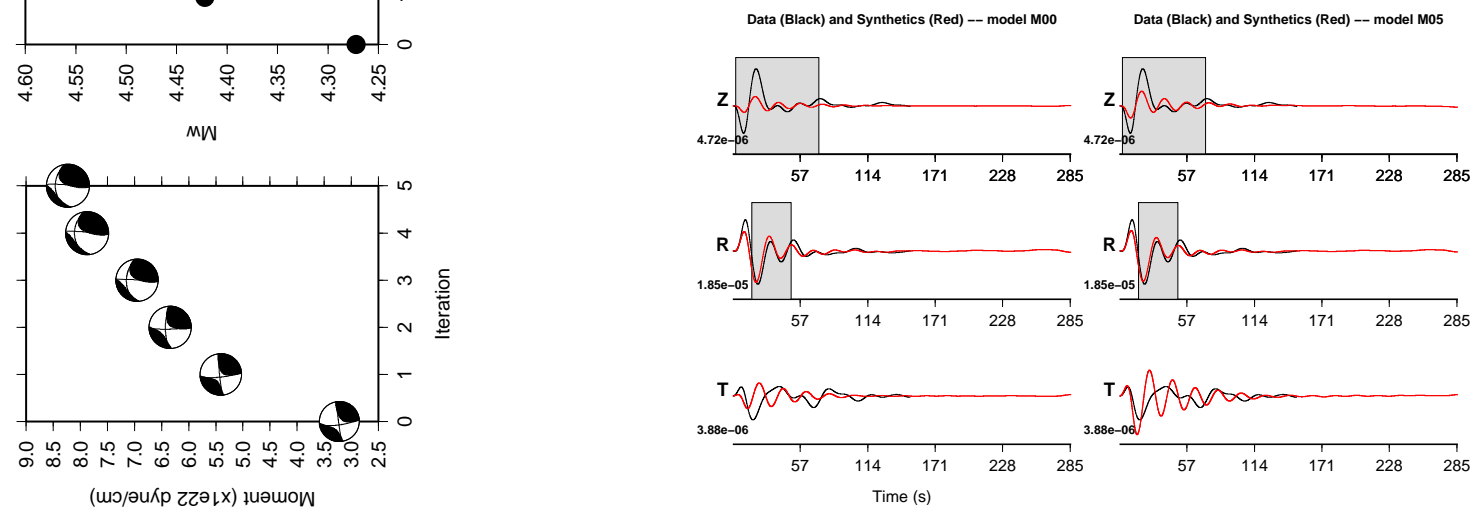

Figure 4: January 4th 2009 event at the Geysers: iterative inversion for the 6 moment tensor coefficients. Each panel displays the scalar moment, magnitude, depth, misfit, and norm of the gradient, from bottom to top. The initial source solution $m_{00}$ is a deviatoric solution from the NCEDC catalog. Convergence is achieved after 5 iterations.

Figure 5: January 4th 2009 event at the Geysers: comparison of the 3-component seismograms at station HOPS between data and the initial model $\mathrm{m}_{00}$ and final model $\mathrm{m}_{05}$. 


\section{Full moment tensor inversion}

\section{REFERENCES}

Dreger, D., and B. Romanowicz, 1994, Source characteristics of events in the san francisco bay region: U.S. Geol. Surv. Open-File Report, 94-176,301-309.

Kim, Y., Q. Liu, and J. Tromp, 2011, Adjoint centroid-moment tensor inversions: Geophys. J. Int., 186, 264-278.

Komatitsch, D., S. Tsuboi, and J. Tromp, 2005, The spectralelement method in seismology, in The Seismic Earth: AGU.

Maggi, A., C. Tape, M. Chen, D. Chao, and J. Tromp, 2009, An automated time window selection algorithm for seismic tomography: Geophys. J. Int., 178, 257-281.

Morency, C., Y. Luo, and J. Tromp, 2009, Finite-frequency kernels for wave propagation in porous media based upon adjoint methods: Geophys. J. Int., 179, 1148-1168.

Pasyanos, M. E., D. S. Dreger, and B. Romanowicz, 1996, Towards real-time estimation of regional moment tensors: Bull. Seismol. Soc. Am., 86, 1255-1269.

Peter, D., B. Savage, A. Rodgers, C. Morency, and J. Tromp, 2011, Adjoint tomography of the middle east: Abstract S44A-04 presented at 2011 Fall Meeting, AGU, San Francisco, Calif., 5-9 Dec.

Tape, C., Q. Liu, A. Maggi, and J. Tromp, 2009, Adjoint tomography of the southern california crust: Science, 325, 988-992.

Tromp, J., C. H. Tape, and Q. Liu, 2005, Seismic tomography, adjoint methods, time reversal, and banana-doughnut kernels: Geophys. J. Int., 160, 195-216. 